\title{
Characteristics and clinical relevance of late gadolinium enhancement in cardiac magnetic resonance in patients with systemic sclerosis
}

\author{
Makoto Sano · Hiroshi Satoh $\cdot$ Kenichiro Suwa $\cdot$ Mamoru Nobuhara $\cdot$ Takeji Saitoh · \\ Masao Saotome · Tsuyoshi Urushida $\cdot$ Hideki Katoh $\cdot$ Kumiko Shimoyama $\cdot$ Daisuke Suzuki $\cdot$ \\ Noriyoshi Ogawa $\cdot$ Yasuo Takehara $\cdot$ Harumi Sakahara $\cdot$ Hideharu Hayashi
}

Received: 16 February 2014/ Accepted: 13 June 2014/Published online: 5 July 2014

(C) The Author(s) 2014. This article is published with open access at Springerlink.com

\begin{abstract}
Cardiac involvement in systemic sclerosis (SSc) is considerably frequent in autopsy, but the early identification is clinically difficult. Recent advantages in cardiac magnetic resonance (CMR) enabled to detect myocardial fibrotic scar as late gadolinium enhancement (LGE). We aimed to examine the prevalence and distribution of LGE in patients with SSc, and associate them with clinical features, electrocardiographic abnormalities and cardiac function. Forty patients with SSc (58 \pm 14 years-old, 35 females, limited/diffuse 25/15, disease duration $106 \pm 113$ months) underwent serological tests, 12-lead electrocardiogram (ECG) and CMR. Seven patients $(17.5 \%)$ showed LGE in 26 segments of left ventricle (LV). LGE distributed mainly in the basal to mid inter-ventricular septum and the right ventricular (RV) insertion points, but involved all the myocardial regions. More patients with LGE showed NYHA functional class II
\end{abstract}

M. Sano $\cdot$ H. Satoh $(\bowtie) \cdot$ K. Suwa $\cdot$ M. Nobuhara .

M. Saotome · T. Urushida $\cdot$ H. Katoh $\cdot$ H. Hayashi

Division of Cardiology, Internal Medicine III, Hamamatsu

University School of Medicine, 1-20-1 Handayama,

Higashi-ward, Hamamatsu 431-3192, Japan

e-mail: satoh36@hama-med.ac.jp

T. Saitoh

Department of Emergency Medicine, Hamamatsu University

School of Medicine, Hamamatsu, Japan

K. Shimoyama $\cdot$ D. Suzuki $\cdot$ N. Ogawa

Division of Immunology and Rheumatology, Internal Medicine

III, Hamamatsu University School of Medicine,

Hamamatsu, Japan

Y. Takehara $\cdot$ H. Sakahara

Department of Radiology, Hamamatsu University School

of Medicine, Hamamatsu, Japan and more (71 vs. $21 \%, p<0.05$ ), bundle branch blocks (57 vs. $6 \%, p<0.05), \quad \mathrm{LV}$ ejection fraction (LVEF) $<50 \%$ (72 vs. $6 \%, p<0.01$ ), LV asynergy (43 vs. $0 \%, p<0.01)$ and $\mathrm{RVEF}<40 \%$ (100 vs. $39 \%$, $p<0.01)$. There was no difference in disease duration, disease types, or prevalence of positive autoimmune antibodies or high serum NT-proBNP level $(>125 \mathrm{pg} / \mathrm{ml})$. When cardiac involvement of SSc was defined as low LVEF, ECG abnormalities or high NT-proBNP, the sensitivity, specificity positive and negative predictive values of LGE were 36, 92, 71 and $72 \%$, respectively. We could clarify the prevalence and distribution of LGE in Japanese patients with SSc. The presence of LGE was associated with cardiac symptom, conduction disturbance and impaired LV/RV contraction.

Keywords Electrocardiography · Conduction disturbance - Cardiac function - Systemic scleroderma . Late gadolinium enhancement

\section{Introduction}

Systemic sclerosis (SSc) is characterized by vascular changes and fibrosis of the skin and internal organs. The prevalence of cardiac involvement in SSc was considered clinically to be $1.4-5.4 \%$ for impaired left ventricular ejection fraction (LVEF), or $18-30 \%$ for diastolic dysfunction [1-3]. While in autopsy, myocardial fibrosis was identified in 50-80\% [4, 5]. In some pathological reports, cardiac involvement in SSc was assumed to be derived from impairment of the microcirculation and primary myocardial fibrosis, and from ischemic damage due to coronary atherosclerosis [6-8]. Patients with cardiac involvement have a poor prognosis because of the 
congestive heart failure and fatal arrhythmias associated with conduction disturbance [9].

Unfortunately, especially in the early phase, most patients with cardiac involvement are asymptomatic and difficult to be detected in subclinical stage. Previous studies have suggested that tissue Doppler echocardiography is useful for detection of the depressed contractility [3], and serum N-terminal-pro brain natriuretic peptide (NT-proBNP) can be a surrogate marker of cardiac involvement [10]. Recently, the values of cardiac magnetic resonance $(\mathrm{CMR})$ are suggested for the early detection of cardiac involvement in SSc. Cine-CMR can assess cardiac morphology and function with high spatial resolution, and late gadolinium enhancement (LGE)-CMR can differentiate fibrotic scar from normal myocardium [11, 12]. Actually, in reports from Western countries, LGE was observed in 21-66\% of patients with SSc [13-15]. However, there are no such data in Asian patients, and only few studies have examined the values of LGE by comparison with echocardiographic findings and serum NT-proBNP level [13, 14].

This study aimed to assess the prevalence and distribution of LGE in patients with SSc, and to associate them with clinical features, electrocardiographical abnormalities and cardiac function.

\section{Patients and methods}

\section{Patients}

This was a single center trans-sectional study. We selected a total of 47 consecutive patients with SSc (17-77 years old) attending the division of immunology and rheumatology at Hamamatsu University Hospital between January 2012 and March 2013. The diagnosis of SSc was based on the guideline of Japanese Ministry of Health and Welfare [16], and the type of SSc was classified into limited or diffuse type according to the LeRoy's classification [17]. Patients were excluded if they had (1) the history of coronary arterial disease, severe valvular diseases or obvious cardiomyopathies, (2) renal insufficiency with an estimated GFR $<30 \mathrm{ml} / \mathrm{min} / 1.73 \mathrm{~m}^{2}$, (3) implanted pacemaker, or (4) no informed consent. Finally, a total of 40 patients were enrolled in this study.

All patients underwent CMR, serological test and 12-lead electrocardiogram (ECG) within 1 month. The serological test included cardiac biomarkers such as NTproBNP and troponin I, and autoimmune antibodies such as anti-Scl-70, anti-centromere and anti-U1-RNP. This study protocol was conducted in accordance with the Declaration of Helsinki and was approved by an institutional review board. All patients gave their informed consent.
CMR protocol

CMR imaging was performed on a 1.5 tesla (T) MR system (Signa Infinity Twinspeed, GE Medical Systems, Waukesha, USA) with a gradient system performance of maximum amplitude of $40 \mathrm{mT} / \mathrm{m}$ and slew time of $150 \mathrm{~T} / \mathrm{m} / \mathrm{s}$ [18]. An 8-element phased array cardiac coil was used in all studies. Three planes such as short axis, sagittal long axis and 4-chamber view were obtained for 2-dimensional (2D) FIESTA cine images and LGE images. The slice thickness/gap was typically $10 \mathrm{~mm} / 0 \mathrm{~mm}$ (6-9 slices). Breath-hold cine magnetic resonance images were obtained in contiguous short-axis planes from apex to base of the heart with the patient in a resting state. The 2D FIESTA cine images were based on the steady state free precession sequence. The imaging parameters were as follows; matrix of $192 \times 192$, field of view of $34 \mathrm{~cm}$, flip angle of $45^{\circ}$, and readout bandwidth of $125 \mathrm{kHz}$. Sixteen data lines were acquired per each segment. The shortest repetition time and echo time were selected; however, the values were not exactly the same for each study, because they were related to the orientation of the scanning plane and slice thickness.

Late gadolinium enhancement images were acquired from $15 \mathrm{~min}$ after an injection of $0.2 \mathrm{mmol} / \mathrm{kg}$ of contrast material (Gd-DTPA-BMA, Fuji Pharma., Tokyo, Japan). LGE imaging was based on the inversion recovery prepared fast gradient echo (IR-FGRE) sequence. The imaging parameters were as follows; matrix of $256 \times 160$, field of view of $34 \mathrm{~cm}$, flip angle of $20^{\circ}$, readout bandwidth of $31.25 \mathrm{kHz}$. The IR-FGRE technique repeated during every R-to-R interval and the trigger delay was $300 \mathrm{~ms}$. The readout data line was 160 each, where 24 data lines were acquired per segment. The inversion time (200-240 ms) was individually determined right before the LGE imaging on basis to optimize nulling of the normal myocardium signal. The process to identify optimum contrast was concluded within 3 min.

\section{Analysis of CMR}

Two experienced cardiovascular radiologists (M.S. and H.S.) interpreted all the CMR images without any knowledge of clinical findings. The 17-segments model was used for segmental analyses for morphology, function and LGE. LV/RV end-diastolic volume (LVEDV/RVEDV), endsystolic volume (LVESV/RVESV) and LV/RVEF were acquired from 2D FIESTA cine images in the short axis view. For $\mathrm{LV} / \mathrm{RV}$ volume analysis, both the endocardial and epicardial contours for LV and only the endocardial contour for RV were manually traced in both end-diastole and end-systole phase, using analysis software (AW VolumeShare $2^{\mathrm{TM}}$, GE Medical Systems, Waukesha, USA). All the aortic, pulmonary and tricuspid valve rings were 
excluded from the volume. The LV and RV volume indices (LVEDVI/LVESVI and RVEDVI/RVESVI), and LV mass index (LVMI) were calculated by dividing them with body surface area. Asynergy in LV wall was also determined when several contiguous segments showed reduced contractility compared with other segments. LGE was defined as an area with a signal intensity which was higher than a signal intensity value $>2$ SD above the normal myocardium, and was present in the same myocardial segment in at least two different planes. The presence, location, and pattern of myocardial LGE were determined by the consensus of the two observers. To assess LGE quantitatively, all the short-axis slices from base to apex were inspected visually, and in each image, the boundaries of LGE area were manually traced. The summed LGE area was rendered to LGE volume and the percentage against total muscle volume (\%LGE volume) was calculated [18].

The pericardial involvement was also examined using cine- and LGE-CMR images. We evaluated the presence of pericardial effusion, and the grade of pericardial LGE as shown in constrictive pericarditis [19]. The presence of pericardial effusion and the grade of pericardial LGE were also determined by the consensus of the two observers.

\section{Statistical analyses}

All the data were expressed as the mean \pm standard deviation (SD) of the indicated numbers $(n)$ or percentages, as appropriate. Categorical variables were compared between the patient groups by Chi-square or Fisher exact tests. Continuous variables between groups were examined by unpaired $t$ test. Correlations between numerical parameters were evaluated by Pearson's correlation. The differences were considered to be significant when $p<0.05$. All the statistical analyses were performed using the software IBM SPSS (Ver.21).

\section{Results}

Prevalence and distribution of LGE

We found 26 segments with LGE at the LV myocardium in $7(17.5 \%)$ patients with SSc. The representative three cases with myocardial LGE are shown in Figs. 1, 2 and 3. In general, LGE distributed mainly in the mid-myocardial wall of basal to mid-IVS but involved all myocardial regions. The intra-LV distribution of LGE was 11 IVS (including 1 anterior RV insertion point), 3 anterior, 6 lateral, 4 inferior, and 2 apical wall, respectively. The intramural distribution of LGE was 8 sub-epicardial, 13 midmyocardial, and 5 sub-endocardial wall, respectively. The patterns of LGE were 23 striated and 3 patchy types. The mean \%LGE volume was $2.7 \%$ (range $0.7-7.9 \%$ ). The intra- and inter-observer variability for measurement of $\%$ LGE volume were acceptable (intra-observer $r=0.98$, $p<0.001$, inter-observer $r=0.98, p<0.001$ ). There was no significant correlation between \%LGE volume and LVEF, RVEF, LVEDVI or RVEDVI (data not shown). No LGE was found in RV or atrial wall.

\section{LGE and clinical features}

Table 1 shows general and immunological features in patients with SSc. There was no difference between patients with and without LGE in terms of age, sex, disease duration, disease type, prevalence of systemic hypertension, interstitial pneumonia, renal dysfunction or autoimmune antibodies. In overlaps of other autoimmune diseases, patients with LGE had 1 polymyositis (PM) and 1 chronic thyroiditis (CT), while patients without LGE had 3 systemic lupus erythematosus (SLE), 4 PM, 1 rheumatoid arthritis (RA), 1 polyarteritis nodosa (PN), and 4 CT. Medications were mainly corticosteroids, immunosuppressors and prostanoids. Calcium blockers, angiotensinconverting enzyme inhibitor and angiotensin II receptor blockers were not widely administered. The prevalence of other autoimmune diseases and medications did not differ between patients with and without LGE.

Table 2 demonstrates cardiac features in all patients. Five patients $(71 \%)$ with LGE had New York Heart Association (NYHA) classes $\geq \mathrm{II}$, whereas nine $(27 \%)$ without LGE did $(p<0.05)$. Four patients with LGE showed high serum levels of NT-proBNP $(>125 \mathrm{pg} / \mathrm{ml})$, but seven without LGE also had, and the difference did not reach significant ( 57 vs. $21 \%, p=$ ns.). In 12-lead ECG, 4 patients $(57 \%)$ with LGE showed bundle branch blocks (see Fig. 1), whereas only three patients without LGE $(9 \%)$ did $(p<0.05)$. Only one patient with LGE showed paroxysmal atrial fibrillation (Case 7). Three patients with LGE had normal ECG (see Figs. 2, 3).

In cine-CMR, five patients with LGE showed low LVEF $(<50 \%)$ and three of them had asynergic wall motion in segments with LGE $(p<0.05$, see Fig. 2). However, two patients without LGE also showed low LVEF. Additionally, all patients with LGE showed RVEF $<40 \%$, whereas 13 patients (39\%) without LGE did. Case 11 (Fig. 4) is a 69-year-old female who was NYHA class II with high NT-proBNP level. She had first degree atrioventricular block, right bundle branch block and abnormal $\mathrm{Q}$ waves. She also showed LV dilatation and globally impaired LV contraction, but had no LGE in myocardium.

The pericardial effusion was observed in three patients (43\%) with LGE and four (12\%) without LGE. Mild to severe pericardial LGE was seen in four patients $(57 \%)$ with LGE and $23(70 \%)$ without LGE. No patient 
Fig. 1 A representative case of $\mathrm{SSc}$ with LGE. Case 1 is a 67-year-old female patient who was NYHA class III with high NT-proBNP level (1,523 pg/ $\mathrm{ml})$. She had complete right bundle branch block with left axis deviation and non-specific ST-T abnormalities in 12-lead ECG (a). Cine-CMR showed normal LV volume and function (b). LGE-CMR exhibited striated and patchy types of LGE distributed in the midmyocardium of anterior RV insertion point and inferior $\mathrm{LV}$ wall (c) (arrows)

A

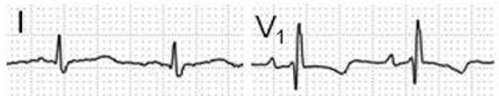

II

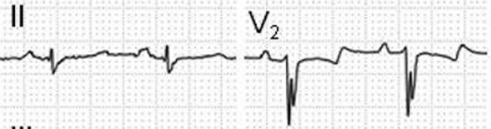

III $\mathrm{V}_{3}$
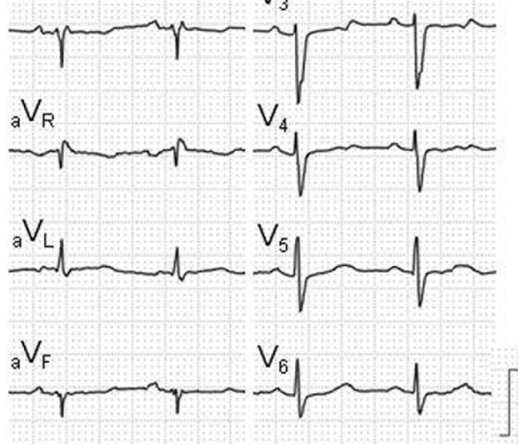

B

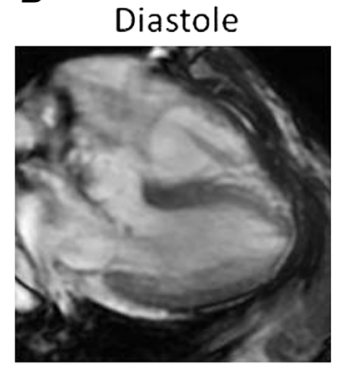

C 4-chamber

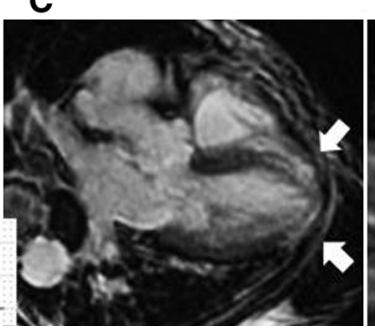

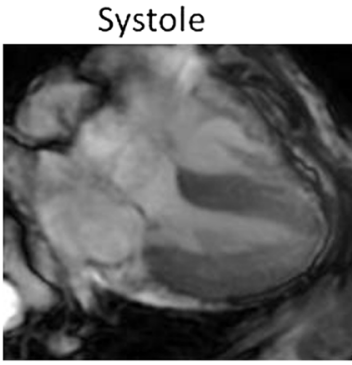

Short-axis

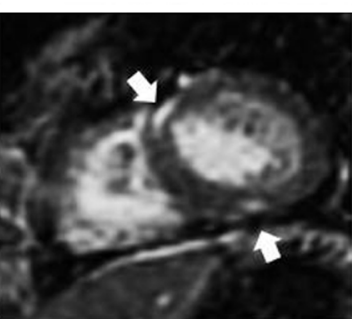

Fig. 2 A representative case of SSc with LGE. Case 2 is a 47-year-old male who was asymptomatic and showed normal ECG, but had high NTproBNP level (196 pg/ml) (a). Cine- and LGE-CMR showed asynergic wall motion and wall thinning (circle) with patchy LGE (arrows) in apical septum (b and $\mathbf{c}$ )

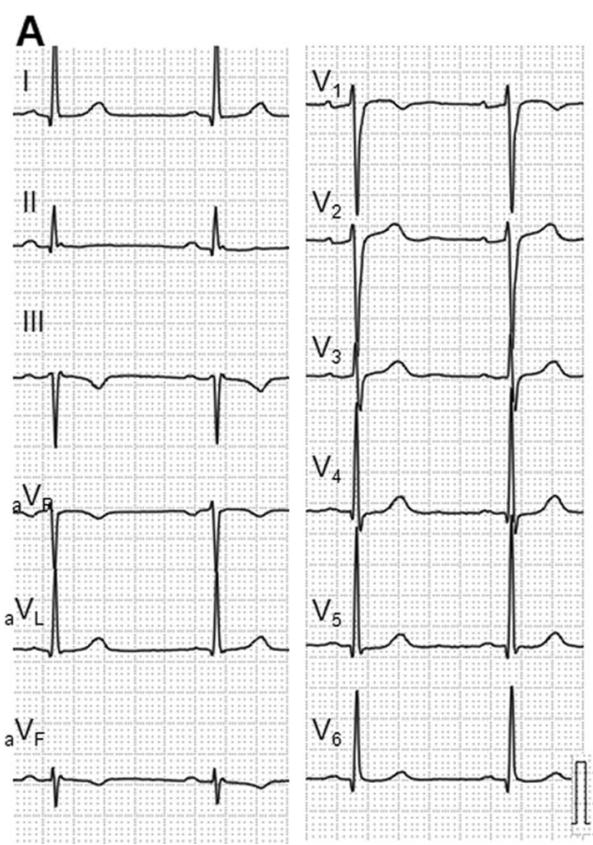

B
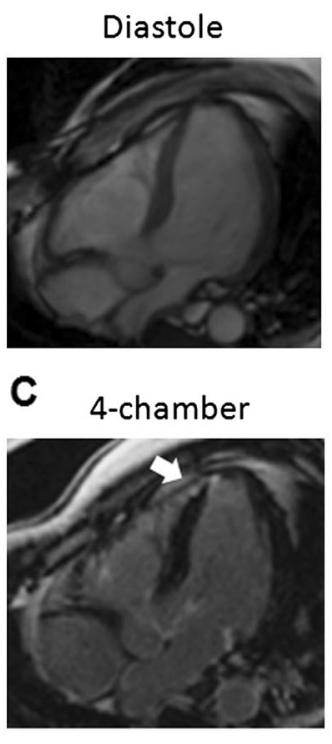

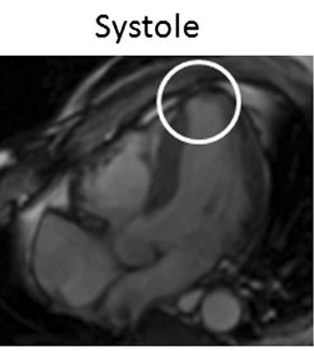

Short-axis

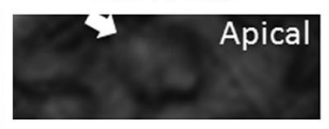

Mid demonstrated pericardial thickness over $4 \mathrm{~mm}$. The prevalence of pericardial effusion or LGE did not differ between patients with and without LGE (86 vs. $76 \%$, $p=$ ns.).

Diagnostic values of LGE for cardiac involvement of SSc

When cardiac involvement of SSc was defined as low LVEF, ECG abnormalities or high NT-proBNP (except patients with eGFR $<60 \mathrm{ml} / \mathrm{min} / 1.73 \mathrm{~m}^{2}$ ), the sensitivity and specificity of LGE were 36 and $92 \%$, and positive and negative predictive values were 71 and $72 \%$, respectively.

\section{Discussion}

This study examined CMR in patients with SSc and showed that (1) LGE in myocardium was considerably frequent, (2) LGE distributed mainly in the basal to mid IVS and the RV insertion points, but involved all myocardial regions, and (3) more patients with LGE were 
Fig. 3 A representative case of SSc with LGE. Case 6 is a 31-year-old female patient who was asymptomatic and had normal NT-proBNP level (33 pg/ml) and normal ECG (a). Cine-CMR showed normal LV volume and function (b). LGECMR exhibited striated type of LGE distributed in the midmyocardium of basal and mid LV wall (c) (arrows). The circumferential pericardial LGE was also apparent
A

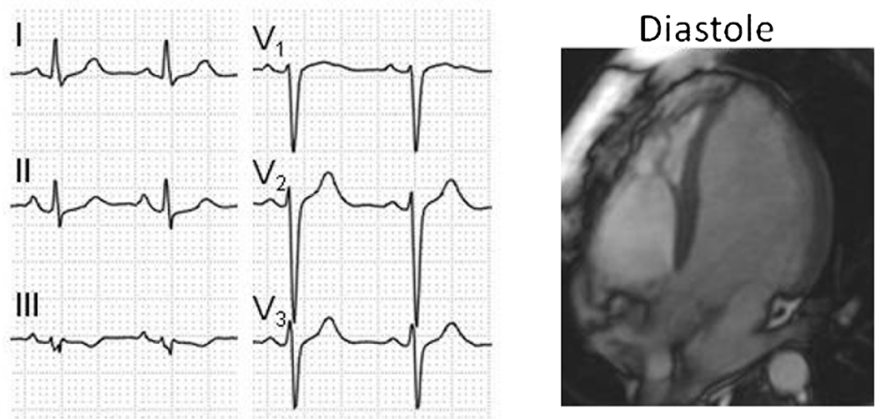

C Long-axis

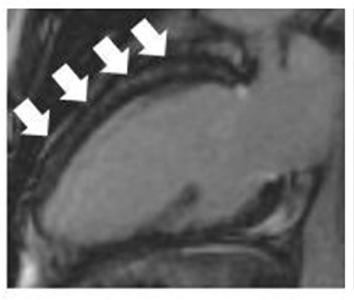

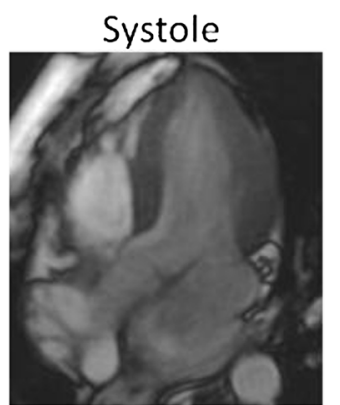

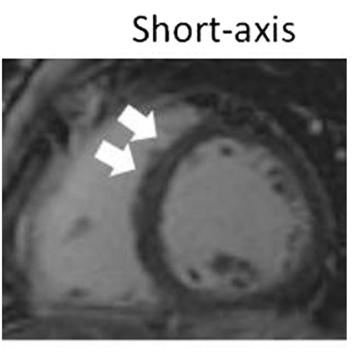

Table 1 General and immunological features in patients with and without LGE

\begin{tabular}{|c|c|c|c|}
\hline & $\operatorname{LGE}(+)$ & $\operatorname{LGE}(-)$ & $p$ value \\
\hline Number & 7 & 33 & \\
\hline Age (y.o.) & $54.0 \pm 22.8$ & $59.3 \pm 11.0$ & ns. \\
\hline Female $(\%)$ & $6(86 \%)$ & $29(88 \%)$ & ns. \\
\hline Disease duration (months) & $49.7 \pm 38.2$ & $118.3 \pm 120.5$ & ns. \\
\hline $\begin{array}{l}\text { Disease type (limited/ } \\
\text { diffuse) }\end{array}$ & $3 / 4$ & $11 / 22$ & ns. \\
\hline Systemic hypertension & $1(14 \%)$ & $10(30 \%)$ & ns. \\
\hline Interstitial pneumonia & $3(43 \%)$ & $15(45 \%)$ & ns. \\
\hline $\begin{array}{l}30 \leq \mathrm{eGFR}<60 \mathrm{ml} / \mathrm{min} / \\
1.73 \mathrm{~m}^{2}\end{array}$ & $0(0 \%)$ & $6(18 \%)$ & ns. \\
\hline \multicolumn{4}{|l|}{ Autoimmune antibodies } \\
\hline Anti-Scl-70 antibody & $1(14 \%)$ & $4(12 \%)$ & ns. \\
\hline Anti-centromere antibody & $2(29 \%)$ & $10(30 \%)$ & ns. \\
\hline Anti-U1-RNP antibody & $1(14 \%)$ & $8(24 \%)$ & ns. \\
\hline \multicolumn{4}{|l|}{ Overlaps } \\
\hline SLE/PM/RA/PN/CT & $0 / 1 / 0 / 0 / 1$ & $3 / 4 / 1 / 1 / 4$ & ns. \\
\hline Medications & & & ns. \\
\hline Corticosteroids & $4(57 \%)$ & $12(36 \%)$ & ns. \\
\hline Immunosupressors & $1(14 \%)$ & $6(18 \%)$ & ns. \\
\hline Prostanoids & $4(57 \%)$ & $11(33 \%)$ & ns. \\
\hline Calcium blockers & $1(14 \%)$ & $4(12 \%)$ & ns. \\
\hline $\mathrm{ACEI} / \mathrm{ARBs}$ & $0(0 \%)$ & $10(30 \%)$ & ns. \\
\hline
\end{tabular}

The categorical variables were expressed as number and percentage (\%) and compared by Chi square test. The continuous variables were expressed as mean $\pm \mathrm{SD}$ and examined by unpaired $t$ test

$A C E I$ angiotensin converting enzyme inhibitors, $A R B$ angiotensin receptor blockers, $C T$ chronic thyroiditis, $e G F R$ estimated glomerular filtration rate, $P M$ polymyositis, $P N$ polyarteritis nodosa, $R A$ rheumatoid arthritis, SLE systemic lupus erythematosus, $n s$ not significant symptomatic and had ECG abnormalities, low LV/RV EF and asynergy. We could clarify, for the first time, the prevalence and distribution of LGE, and the association with ECG abnormalities and impaired cardiac function in patients with SSc.

\section{Myocardial fibrosis and LGE in SSc}

In autopsy, myocardial fibrosis in SSc was identified in 50-80\% [4, 5]. Bulkley et al. [5] reported that myocardial lesions in SSc consisted of contraction band necrosis, focal fibrotic changes, and muscle cell necrosis, and replacement fibrosis. The focal fibrosis was randomly localized throughout the layers of myocardium both in RV and LV. In endomyocardial biopsy, patients with SSc had more interstitial collagen volume fraction than normal controls regardless of signs of heart failure [20]. However, the endomyocardial biopsy is an invasive manner, and it has been in demand for non-invasive evaluation of fibrotic lesion.

This study showed that LGE has been distributed in 7 (17.5\%) of 40 Japanese patients with SSc. In previous reports from Western countries, LGE was observed in 21-66 \% [13-15]. The difference in the prevalence of LGE might result from variations in the patient populations including racial differences.

Mechanisms of fibrosis and LGE in SSc

In pathological studies, myocardial fibrosis in SSc was assumed to be derived from impairment of 


\section{: \\ 幽

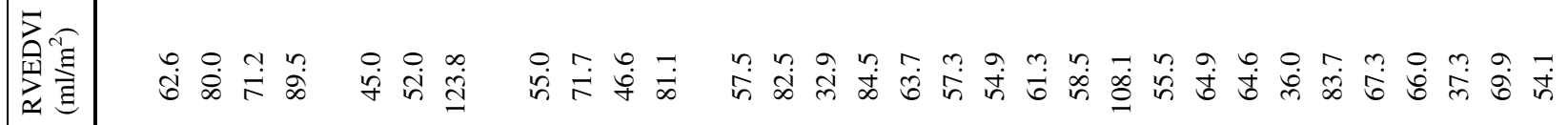 荘

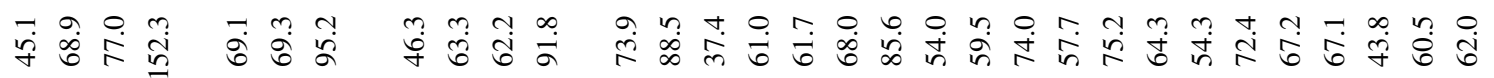

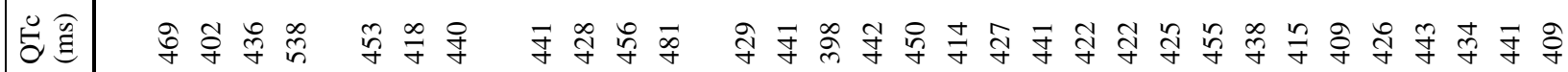

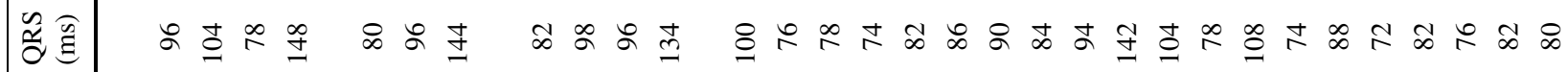

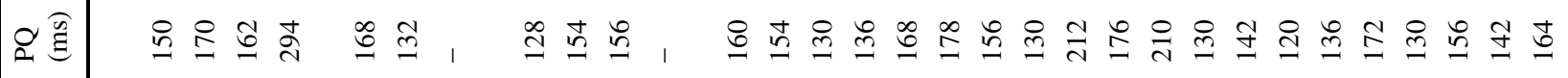

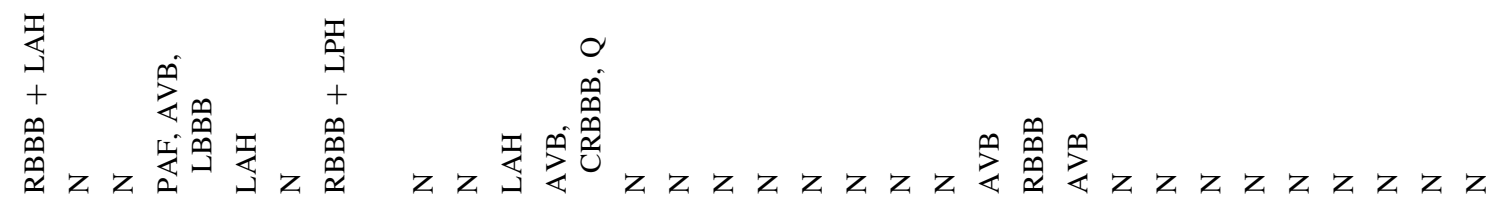

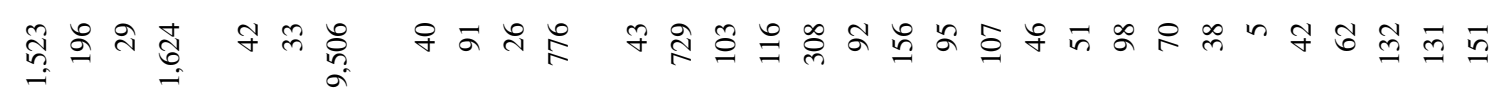

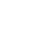




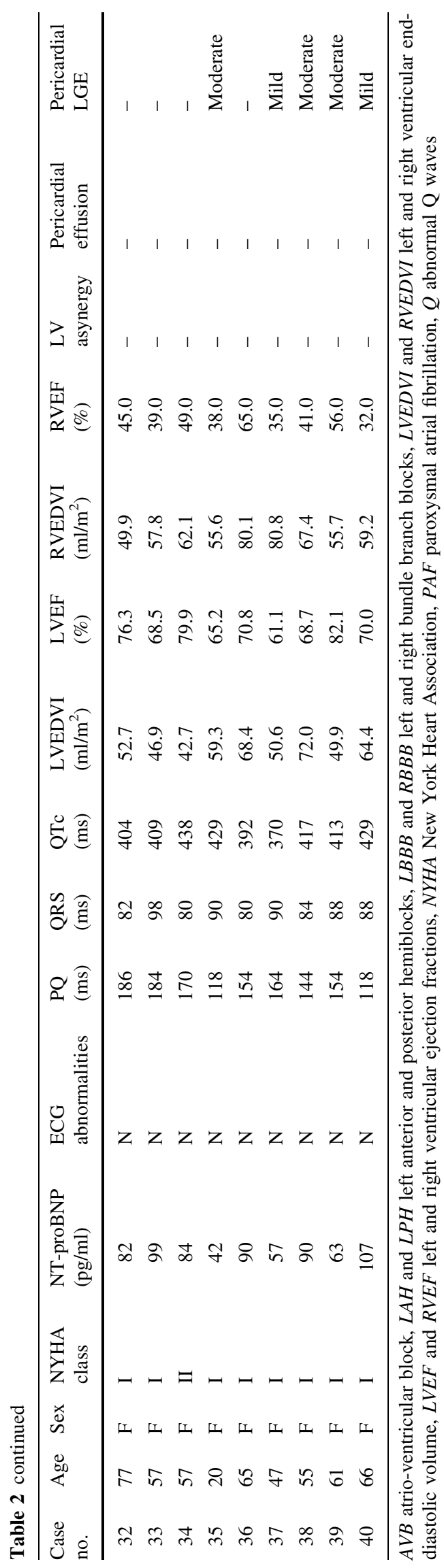

microcirculation due to micro-vascular abnormalities or primary myocardial fibrosis, and from ischemic damage due to coronary atherosclerosis [6-8]. In addition, mononuclear cell infiltration, mainly consisted of CD-3 positive $\mathrm{T}$ cells, has been reported in myocardial biopsies [7, 21]. In our findings, LGE distributed mainly in the basal to midIVS, but involved all myocardial layers. Although LGE regions involved sub-endocardial layers, the patchy types of distribution and discordance with certain coronary perfusion areas might exclude the myocardial infarction due to coronary atherosclerosis. Tzelepis et al. [13] also reported that all regions with LGE were localized in the basal segments and exhibited linear pattern in the mid-myocardial layer with spared sub-endocardium. Hence, LGE in SSc is likely to be caused by various etiologies including dilated cardiomyopathy (DCM)-like mid-wall fibrosis, inflammation, and ischemia $[22,23]$. The additional analysis with T2-weighted CMR may help to clarify the intimate mechanism of LGE in patients with SSc [24].

However, although LGE-CMR can differentiate the myocardial fibrotic scar from normal myocardium [11], diffuse fibrosis cannot be visualized, because myocardium with diffuse fibrosis was "nulled" to highlight focal scar [25]. In the present study, a small number of patients without LGE showed low LVEF, suggesting undiagnosed LV wall damage in such patients.

Additionally, several studies have shown that patients with pulmonary arterial hypertension have LGE in the RV insertion points and in IVS [26], and that the presence of LGE correlates with RV dysfunction and poor prognosis $[27,28]$. In this study, two patients had LGE in the RV insertion points, and all patients with LGE exhibited low RVEF [29]. Hesselstrand et al. [30] reported that LGE in the RV insertion points is a characteristic feature of connective tissue disease-related pulmonary arterial hypertension, although the mechanism is unknown.

\section{Clinical relevance of LGE in SSc}

Many studies have associated LGE with clinical and ECG features and long-term cardiac events in patients with ischemic and non-ischemic cardiomyopathies [22], [3136]. Here we examined whether LGE might be a surrogate marker of cardiac involvement in SSc. We showed that more patients with LGE were symptomatic (NYHA classes $\geq$ II), although there was no difference in the prevalence of high serum NT-proBNP level. The patients without LGE included those with low eGFR might affect the negative result (see Table 1). Allanore et al. [10] reported that serum NT-proBNP level can be a surrogate marker of cardiac involvement in SSc.

The presence of LGE was not associated with disease duration, disease types, or autoimmune antibodies. 
Fig. 4 A representative case of SSc without LGE. Case 11 is a 69-year-old female who was NYHA class II with high NTproBNP level $(776 \mathrm{pg} / \mathrm{ml})$. She had first degree atrio-ventricular block, right bundle branch block and abnormal Q waves (a). Cine-CMR showed LV dilatation and globally impaired $\mathrm{LV}$ contraction $(\mathrm{LVEF}=44 \%$, (b), but LGE-CMR demonstrated no LGE in myocardium (c). The circumferential pericardial LGE was also apparent

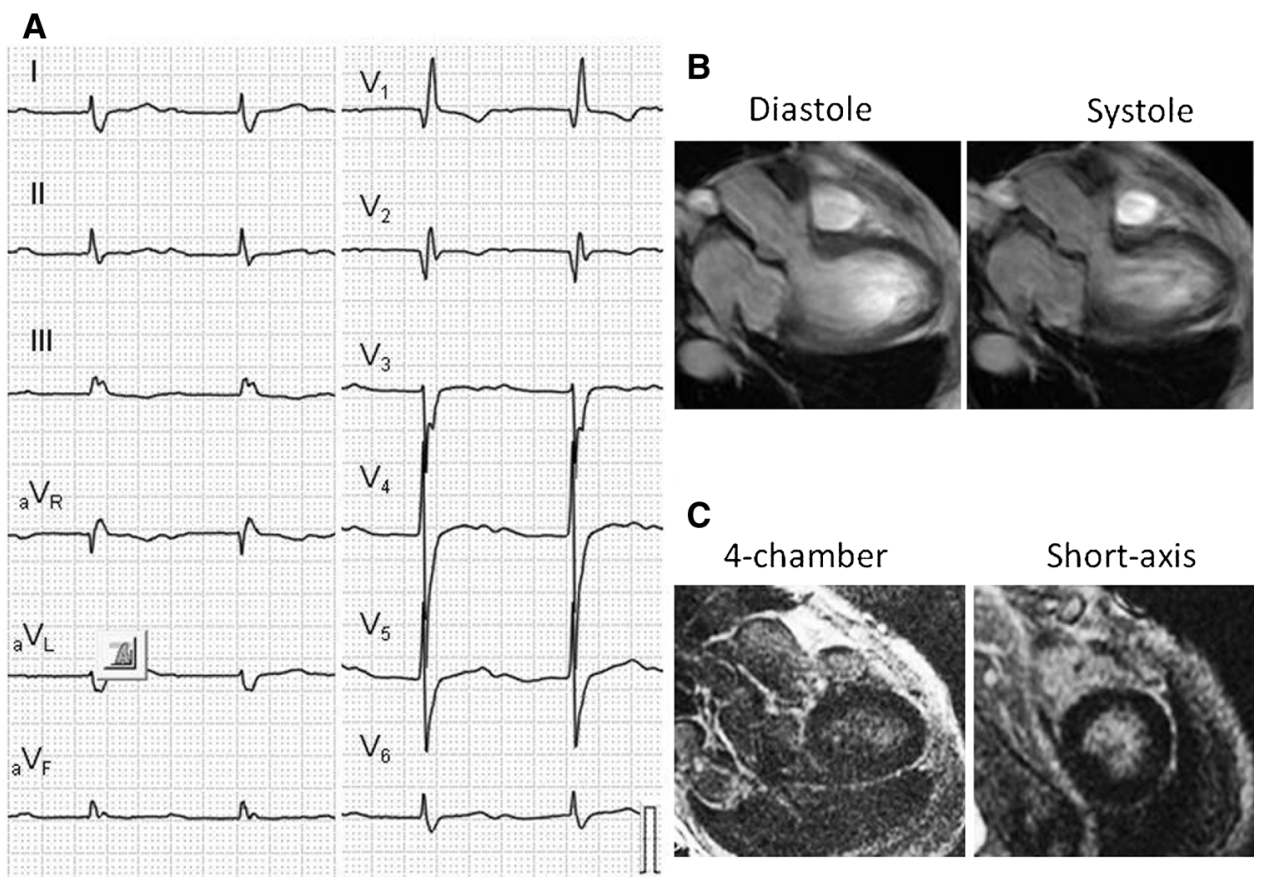

However, Tzelepis et al. [13] showed a close relationship between LGE volume and the duration of Raynaud's phenomenon, and Steen et al. [37, 38] reported significant correlations of anti-Scl 70 and anti-centromere antibodies with organ involvements.

In 12-lead ECG, more patients with LGE had bundle branch blocks. Follansbee et al. [39] reported that in 102 patients with SSc, ventricular conduction abnormalities were present in $17 \%$. The finding that LGE was mainly localized in IVS might be associated with higher prevalence of conduction disturbance.

In cardiac function, previous reports showed that even asymptomatic patients with SSc had systolic and diastolic dysfunction in echocardiography [40-42]. In this study, more patients with LGE had low LVEF, LV asynergy, and low RVEF. However, there were no significant correlations between \%LGE volume and LV/RV EDVI and EF. The reason was uncertain but a limitation of LGE-CMR in terms of estimation of diffuse fibrosis might cause the negative results. Furthermore, two patients with LGE showed normal NT-proBNP level, ECG, and LVEF. Thus, the ability of LGE-CMR to detect cardiac fibrosis in the subclinical stage may help identification of high risk patients and early initiation of therapeutic interventions, although the relevance in long term prognosis remains to be elusive.

Finally, the pericardial involvement was frequent in our patients with SSc, although the prevalence did not differ between patients with and without myocardial LGE. Previous necropsy studies showed pericardial diseases in $33-77 \%$ of the cases, whereas any symptoms occurred in only $7-20 \%$. The pericardial involvement included fibrinous pericarditis, chronic fibrous pericarditis, pericardial adhesions, and pericardial effusions [5, 43]. We can also show the usefulness of pericardial imaging with CMR for the detection of pericardial involvement of SSc in asymptomatic patients.

Limitations

First, as mentioned above, LGE-CMR cannot visualize diffuse fibrosis, because myocardium with diffuse fibrosis is regarded as normal by the nulling method. Actually, the sensitivity of LGE was low for cardiac involvement of SSc defined with other diagnostic modalities. However, previous studies and our data suggest the analysis of fibrotic scar with LGE-CMR still has clinical relevance [22, 33, 34]. This disadvantage might be referred to DCM, although novel T1 mapping techniques can quantitatively assess myocardial fibrosis [25]. Second, coronary angiography and endomyocardial biopsy were not routinely performed to exclude coronary arterial disease and idiopathic/secondary cardiomyopathies. However, both examinations are invasive and the use of contrast media should be avoided especially in patients with reduced renal function. Finally, small sample size and number of patients with LGE and the lack of prognostic evaluation might cause negative results for some clinical comparisons, and be limitations for extrapolating our data to diverse groups of patients. We did not compare the continuous variables for cardiac features, but just examined the prevalence of abnormal findings. 


\section{Conclusions}

This study could clarify the prevalence and distribution of LGE in patients with SSc. The presence of LGE was associated with cardiac symptom, conduction disturbance and impaired LV and RV contraction. Further studies are necessary to elucidate the relevance of LGE for early detection of cardiac involvement and for prediction of long term outcomes in patients with SSc.

\section{Conflict of Interest None.}

Open Access This article is distributed under the terms of the Creative Commons Attribution License which permits any use, distribution, and reproduction in any medium, provided the original author(s) and the source are credited.

\section{References}

1. de Groote P, Gressin V, Hachulla E, Carpentier P, Guillevin L, Kahan A, Cabane J, Frances C, Lamblin N, Diot E, Patat F, Sibilia J, Petit H, Cracowski JL, Clerson P, Humbert M (2008) Evaluation of cardiac abnormalities by doppler echocardiography in a large nationwide multicentric cohort of patients with systemic sclerosis. Ann Rheum Dis 67:31-36

2. Allanore Y, Meune C, Vonk MC, Airo P, Hachulla E, Caramaschi P, Riemekasten G, Cozzi F, Beretta L, Derk CT, Komocsi A, Farge D, Balbir A, Riccieri V, Distler O, Chiala A, Papa ND, Simic KP, Ghio M, Stamenkovic B, Rednic S, Host N, Pellerito R, Zegers E, Kahan A, Walker UA, Matucci-Cerinic M (2010) Prevalence and factors associated with left ventricular dysfunction in the EULAR scleroderma trial and research group (EUSTAR) database of patients with systemic sclerosis. Ann Rheum Dis 69:218-221

3. Meune C, Avouac J, Wahbi K, Cabanes L, Wipff J, Mouthon L, Guillevin L, Kahan A, Allanore Y (2008) Cardiac involvement in systemic sclerosis assessed by tissue-doppler echocardiography during routine care: a controlled study of 100 consecutive patients. Arthritis Rheum 58:1803-1809

4. D'Angelo WA, Fries JF, Masi AT, Shulman LE (1969) Pathologic observations in systemic sclerosis (scleroderma). A study of fifty-eight autopsy cases and fifty-eight matched controls. Am J Med 46:428-440

5. Bulkley BH, Ridolfi RL, Salyer WR, Hutchins GM (1976) Myocardial lesions of progressive systemic sclerosis. A cause of cardiac dysfunction. Circulation 53:483-490

6. Kahan A, Allanore Y (2006) Primary myocardial involvement in systemic sclerosis. Rheumatology (Oxford) 45(Suppl 4):14-17

7. Kanno H, Segawa T, Miura Y, Miyata M, Yoshida A, Nakamura M, Sawai T (2011) Systemic sclerosis presented as congestive heart failure: an autopsy case. Mod Rheumatol 21:423-427

8. Nagai Y, Yamanaka M, Hashimoto C, Nakano A, Hasegawa A, Tanaka Y, Yokoo H, Nakazato Y, Ishikawa O (2007) Autopsy case of systemic sclerosis with severe pulmonary hypertension. J Dermatol 34:769-772

9. Ioannidis JP, Vlachoyiannopoulos PG, Haidich AB, Medsger TA Jr, Lucas M, Michet CJ, Kuwana M, Yasuoka H, van den Hoogen F, Te Boome L, van Laar JM, Verbeet NL, Matucci-Cerinic M, Georgountzos A, Moutsopoulos HM (2005) Mortality in systemic sclerosis: an international meta-analysis of individual patient data. Am J Med 118:2-10
10. Allanore Y, Wahbi K, Borderie D, Weber S, Kahan A, Meune C (2009) N-terminal pro-brain natriuretic peptide in systemic sclerosis: a new cornerstone of cardiovascular assessment? Ann Rheum Dis 68:1885-1889

11. Kim RJ, Wu E, Rafael A, Chen EL, Parker MA, Simonetti O, Klocke FJ, Bonow RO, Judd RM (2000) The use of contrastenhanced magnetic resonance imaging to identify reversible myocardial dysfunction. N Engl J Med 343:1445-1453

12. Saji M, Takamisawa I, Iguchi N, Takayama M (2013) Cardiac MRI detected septal and lateral myocardial infarction by alcohol septal ablation through the intermediate artery. Heart Vessel 28:672-676

13. Tzelepis GE, Kelekis NL, Plastiras SC, Mitseas P, Economopoulos N, Kampolis C, Gialafos EJ, Moyssakis I, Moutsopoulos HM (2007) Pattern and distribution of myocardial fibrosis in systemic sclerosis: a delayed enhanced magnetic resonance imaging study. Arthritis Rheum 56:3827-3836

14. Hachulla AL, Launay D, Gaxotte V, de Groote P, Lamblin N, Devos P, Hatron PY, Beregi JP, Hachulla E (2009) Cardiac magnetic resonance imaging in systemic sclerosis: a cross-sectional observational study of 52 patients. Ann Rheum Dis 68:1878-1884

15. Di Cesare E, Battisti S, Di Sibio A, Cipriani P, Giacomelli R, Liakouli V, Ruscitti P, Masciocchi C (2013) Early assessment of sub-clinical cardiac involvement in systemic sclerosis (SSc) using delayed enhancement cardiac magnetic resonance (CE-MRI). Eur J Radiol 82:e268-e273

16. Takehara K, Satoh S. Kuwana M (2007) Guideline for the diagnosis of systemic sclerosis (in Japanese). The Japanese Ministry of Health Labor and Welfare, 1-32, 2007

17. LeRoy EC, Black C, Fleischmajer R, Jablonska S, Krieg T, Medsger TA Jr, Rowell N, Wollheim F (1988) Scleroderma (systemic sclerosis): classification, subsets and pathogenesis. J Rheumatol 15:202-205

18. Matoh F, Satoh H, Shiraki K, Saitoh T, Urushida T, Katoh H, Takehara Y, Sakahara H, Hayashi H (2007) Usefulness of delayed enhancement magnetic resonance imaging to differentiate dilated phase of hypertrophic cardiomyopathy and dilated cardiomyopathy. J Card Fail 13:372-379

19. Feng D, Glockner J, Kim K, Martinez M, Syed IS, Araoz P, Breen J, Espinosa RE, Sundt T, Schaff HV, Oh JK (2011) Cardiac magnetic resonance imaging pericardial late gadolinium enhancement and elevated inflammatory markers can predict the reversibility of constrictive pericarditis after antiinflammatory medical therapy: a pilot study. Circulation 124:1830-1837

20. Fernandes F, Ramires FJ, Arteaga E, Ianni BM, Bonfa ES, Mady C (2003) Cardiac remodeling in patients with systemic sclerosis with no signs or symptoms of heart failure: an endomyocardial biopsy study. J Card Fail 9:311-317

21. Liangos O, Neure L, Kuhl U, Pauschinger M, Sieper J, Distler A, Schwimmbeck PL, Braun J (2000) The possible role of myocardial biopsy in systemic sclerosis. Rheumatology (Oxford) 39:674-679

22. Assomull RG, Prasad SK, Lyne J, Smith G, Burman ED, Khan M, Sheppard MN, Poole-Wilson PA, Pennell DJ (2006) Cardiovascular magnetic resonance, fibrosis, and prognosis in dilated cardiomyopathy. J Am Coll Cardiol 48:1977-1985

23. De Cobelli F, Pieroni M, Esposito A, Chimenti C, Belloni E, Mellone R, Canu T, Perseghin G, Gaudio C, Maseri A, Frustaci A, Del Maschio A (2006) Delayed gadolinium-enhanced cardiac magnetic resonance in patients with chronic myocarditis presenting with heart failure or recurrent arrhythmias. J Am Coll Cardiol 47:1649-1654

24. Hen Y, Iguchi N, Machida H, Takada K, Utanohara Y, Sumiyoshi $\mathrm{T}$ (2013) High signal intensity on T2-weighted cardiac magnetic 
resonance imaging correlates with the ventricular tachyarrhythmia in hypertrophic cardiomyopathy. Heart Vessels 28:742-749

25. Flett AS, Hayward MP, Ashworth MT, Hansen MS, Taylor AM, Elliott PM, McGregor C, Moon JC (2010) Equilibrium contrast cardiovascular magnetic resonance for the measurement of diffuse myocardial fibrosis: preliminary validation in humans. Circulation 122:138-144

26. Blyth KG, Groenning BA, Martin TN, Foster JE, Mark PB, Dargie HJ, Peacock AJ (2005) Contrast enhanced-cardiovascular magnetic resonance imaging in patients with pulmonary hypertension. Eur Heart J 26:1993-1999

27. McCann GP, Gan CT, Beek AM, Niessen HW, Vonk Noordegraaf A, van Rossum AC (2007) Extent of mri delayed enhancement of myocardial mass is related to right ventricular dysfunction in pulmonary artery hypertension. Am J Roentgenol 188:349-355

28. Freed BH, Gomberg-Maitland M, Chandra S, Mor-Avi V, Rich S, Archer SL, Jamison EB Jr, Lang RM, Patel AR (2012) Late gadolinium enhancement cardiovascular magnetic resonance predicts clinical worsening in patients with pulmonary hypertension. J Cardiovasc Magn Reson 14:11

29. Pfisterer ME, Battler A, Zaret BL (1985) Range of normal values for left and right ventricular ejection fraction at rest and during exercise assessed by radionuclide angiocardiography. Eur Heart $\mathbf{J}$ 6:647-655

30. Hesselstrand R, Scheja A, Wuttge DM, Arheden H, Ugander M (2011) Enlarged right-sided dimensions and fibrosis of the right ventricular insertion point on cardiovascular magnetic resonance imaging is seen early in patients with pulmonary arterial hypertension associated with connective tissue disease. Scand J Rheumatol 40:133-138

31. Moon JC, McKenna WJ, McCrohon JA, Elliott PM, Smith GC, Pennell DJ (2003) Toward clinical risk assessment in hypertrophic cardiomyopathy with gadolinium cardiovascular magnetic resonance. J Am Coll Cardiol 41:1561-1567

32. Bogaert J, Kalantzi M, Rademakers FE, Dymarkowski S, Janssens S (2007) Determinants and impact of microvascular obstruction in successfully reperfused ST-segment elevation myocardial infarction. Assessment by magnetic resonance imaging. Eur Radiol 17:2572-2580

33. Satoh H, Matoh F, Shiraki K, Saitoh T, Odagiri K, Saotome M, Urushida T, Katoh H, Takehara Y, Sakahara H, Hayashi H (2009)
Delayed enhancement on cardiac magnetic resonance and clinical, morphological, and electrocardiographical features in hypertrophic cardiomyopathy. J Card Fail 15:419-427

34. Lehrke S, Lossnitzer D, Schob M, Steen H, Merten C, Kemmling H, Pribe R, Ehlermann P, Zugck C, Korosoglou G, Giannitsis E, Katus HA (2011) Use of cardiovascular magnetic resonance for risk stratification in chronic heart failure: prognostic value of late gadolinium enhancement in patients with non-ischaemic dilated cardiomyopathy. Heart 97:727-732

35. Patel MR, Cawley PJ, Heitner JF, Klem I, Parker MA, Jaroudi WA, Meine TJ, White JB, Elliott MD, Kim HW, Judd RM, Kim RJ (2009) Detection of myocardial damage in patients with sarcoidosis. Circulation 120:1969-1977

36. Zemrak F, Petersen SE (2011) Late gadolinium enhancement $\mathrm{cmr}$ predicts adverse cardiovascular outcomes and mortality in patients with coronary artery disease: systematic review and meta-analysis. Prog Cardiovasc Dis 54:215-229

37. Steen VD, Powell DL, Medsger TA Jr (1988) Clinical correlations and prognosis based on serum autoantibodies in patients with systemic sclerosis. Arthritis Rheum 31:196-203

38. Steen VD (2005) Autoantibodies in systemic sclerosis. Semin Arthritis Rheum 35:35-42

39. Follansbee WP, Curtiss EI, Rahko PS, Medsger TA Jr, Lavine SJ, Owenes GR, Steen VD (1985) The elctrocardiogram in systemic sclerosis (scleroderma). Study of 102 consecutive cases with functional correlations and review of the literature. Am J Med 79:183-192

40. Poanta L, Dadu R, Tiboc C, Rednic S, Dumitrascu D (2009) Systolic and diastolic function in patients with systemic sclerosis. Eur J Intern Med 20:378-382

41. D'Andrea A, Stisi S, Bellissimo S, Vigorito F, Scotto di Uccio F, Tozzi N, Moscato F, Pezzullo E, Calabro R, Scherillo M (2005) Early impairment of myocardial function in systemic sclerosis: non-invasive assessment by doppler myocardial and strain rate imaging. Eur J Echocardiogr 6:407-418

42. Spethmann S, Dreger H, Schattke S, Riemekasten G, Borges AC, Baumann G, Knebel F (2012) Two-dimensional speckle tracking of the left ventricle in patients with systemic sclerosis for an early detection of myocardial involvement. Eur Heart J Cardiovasc Imaging 13:863-870

43. Plastiras SC, Toumanidis ST (2012) Systemic sclerosis: the heart of the matter. Hellenic J Cardiol 53:287-300 